ORIGINAL ARTICLE

Volume 12 Issue 32020

DOI: $10.21315 /$ eimj2020.12.3.1

ARTICLE INFO

Submitted: $28-04-2020$

Accepted: 13-07-2020

Online: 30-09-2020

\section{Developing a Model for Identification of the Borderline Group in Objective Structured Clinical Exam}

\author{
Rabab Abdel Ra'oof', Shimaa Elaraby', Esam Abdelgawad ${ }^{2}$
}

${ }^{1}$ Medical Education Department, Faculty of Medicine, Suez Canal University, EGYPT

${ }^{2}$ Urology Department, Faculty of Medicine, Suez Canal University, EGYPT

To cite this article: Abdel Ra'oof R, Elaraby S, Abdelgawad E. Developing a model for identification of the borderline group in objective structured clinical exam. Education in Medicine Journal. 2020;12(3):1-10. https://doi.org/10.21315/eimj2020.12.3.1

To link to this article: https://doi.org/10.21315/eimj2020.12.3.1

\begin{abstract}
Many standard setting methods have been developed to determine the cut off score in objective structured clinical exam (OSCE). Evaluators face the challenges of determining borderline group in applying these standard setting methods. Rating the students as borderline was a problematic decision. This study aimed to develop performance descriptors of the borderline students in order to enhance the objectivity of the standard setting methods in OSCE. We conducted a qualitative study to delineate a model for characteristics of borderline group in OSCE. The sample was the examiners who were experienced in the assessment of students in clinical skills. Semi-structured interviews were conducted and a thematic analysis was done. Examiners identified four main themes under which characteristics of borderline group could be listed. Three of them belong to different clinical examination domains, which are gathering patient information, examining patients, communicating with patients, and the fourth theme is considered as personal characteristics. Having said that the identification of borderline group is a subjective process, recognising their characteristics may be helpful in increasing objectivity of making decisions in OSCE, especially in evaluating critical areas like patient safety and communication skills.
\end{abstract}

Keywords: Assessment, Borderline, OSCE, Communicating with patient

Shimaa Elaraby, Medical Education Department, Faculty of Medicine, Suez Canal University, 41522 Round Road, Ismailia, Egypt | Email: shimaa80me@yahoo.com

\section{INTRODUCTION}

Objective structured clinical exam (OSCE) is conducted in many institutions nowadays, and it is used as a standard assessment method in a high stake examination such as the Canadian qualifying examination and The National Board of Medical Examiners in the U.S. (1). The obtained test score from the OSCE checklist is used to make high stake decisions. Standard setting is one of the methods used to set the expected pass or fail cut score in an OSCE station (2).

Standard setting refers to the process of establishing one or more cut scores on examinations. The cut scores divide the distribution of examinees' test performances 
into two or more categories (3). Methods of standard setting are divided into two categories: relative (norm-referenced) and absolute (criterion-referenced) procedures. A relative standard is based on the performance of the test takers as a group. The absolute approach defines the passing score in terms of how many items or tasks have to be performed correctly to pass (4). Absolute method can be divided into empirical and judgemental methods. In empirical method, experts judge every checklist item to set pass score for each item. This method can be proposed mainly by Angoff method. This method has the disadvantages that the judges face the complexity of borderline group (5). Judgemental absolute method is also known as borderline regression method (BRM) is based on global grade and checklist scores of OSCE stations; history taking, physical examination, procedural skills, and health education (2). Setting performance descriptors for borderline group is essential in all methods; however, it is a demanding cognitive task and may impair experts' judgement (6).

In all these types and categories, we cannot ignore the subjective nature of standard setting. In some methods, experts are asked to estimate the probability that a borderline candidate would correctly answer the test items. Others require judges to observe and evaluate students' performance during the examination (6).

Although the definition of minimally competent (borderline) student is straightforward, but implementing this definition is difficult. Asking judges to describe borderline students they have known provides a clear understanding of what it means to be "borderline" and enables group consensus before the beginning of the standard setting work (7).

Till now, there are scarce researches in setting these criteria for identifying the borderline group. However, they are crucial for increasing objectivity and thence the validity and reliability of measuring clinical skills of the students in OSCE stations. Our question is, "what are specific characteristics that describe performance of borderline group in each OSCE station from experts' viewpoints according to the nature of the required task to be performed?" The borderline students are recognised to be the ones who do not harm the public in their practice. So, categorising the characteristics of this group will be a new contribution for making a valid decision in clinical exams.

\section{METHODOLOGY}

\section{Conceptual Framework}

This study aimed to set criteria for minimally competent (borderline) group performance in each OSCE station. Setting characteristics for minimally competent (borderline) group is essential in all methods; however, it is a demanding cognitive task and may affect experts' judgement (6).

Identification of borderline group is essential in many of the standard setting methods. It will affect the reliability of the OSCE results. Setting criteria by experts can help in the process of the borderline group identification.

We conducted this study to set criteria for performance of borderline group for each OSCE station from experts' viewpoints. These criteria once established will enhance the process of visualising borderline group and the credibility of standard setting (Figure 1). 


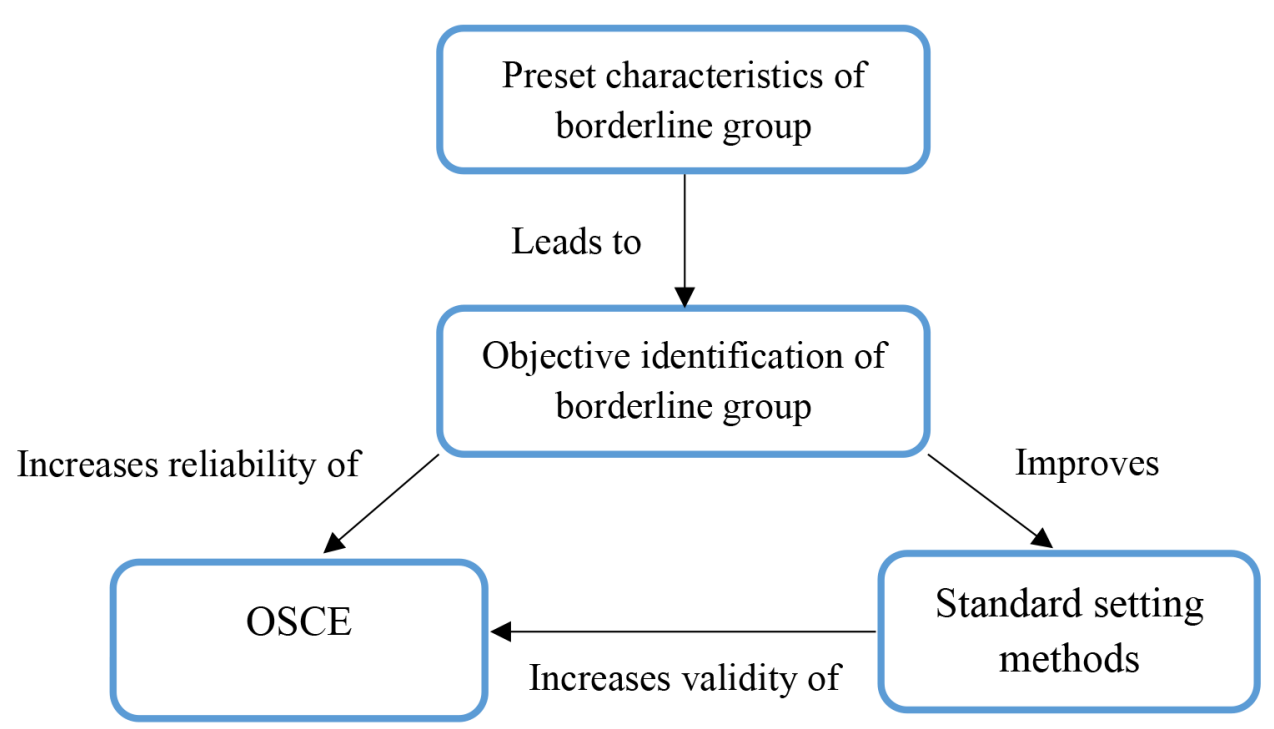

Figure 1: Relationship between characteristics of borderline group, standard setting, reliability and validity of standard setting.

\section{Study Type}

This study is a qualitative phenomenological, where characteristics of borderline group during practical clinical exams were explored from the clinical examiners' viewpoints. Descriptors and model of these characteristics were developed.

\section{Study Population and Sample Type}

It was a non-probability purposeful sample, respondents were a group of experts those have an experience in evaluating students' performance during practical clinical exam in the Faculty of Medicine, Suez Canal University. They had an experience not less than five years, some of them had experience for more than 30 years, but all of them had experience in OSCE not more than three years; because it is a newly implemented method in the institution for clinical exam. Their experience is not necessarily as raters during OSCEs only, but during clinical exams in general.

\section{Sample Size}

Data were collected until there were no new themes or other emerged explanations elicited from data (data saturation), the number of respondents were 12 clinical examiners.

\section{Data Collection Tools}

1. Semi-structured interviews were conducted, predetermined questions were asked during academic year (2017, 2018 and 2018-2019). First, we asked participants about their experiences as clinical examiners. Then, we asked them about their knowledge of standards settings and describe the mechanism of ranking students as competent, borderline and incompetent; what the criteria of such judgement are; and how they find this process.

2. We identified borderline group such as students who have fair performance, who might pass the assessment and on other days might fail (7), and will not harm the population. Then, they need more practicing.

3. We asked them to describe the performance of borderline groups in four main OSCE stations such as history tacking, physical examination, procedural skills, and health education. 
4. Each interview lasted about 30 minutes. It was recorded after having the informed consent and we made transcript for each interview.

\section{Data Analysis}

We transcribed data from recorded interviews and coded it, and then categorised data according to codes, data were presented into themes, which in turn depend on information trends, frequencies, relations, and structure. We supported each theme by quotations from interviews (8). Interviewers do not participate in OSCE as examiners, thus they have no bias regarding interpretation of interviewees' description of borderline groups. Thematic analysis involved listening and re-listening the data records many times, identifying initial themes and subthemes, constructing a hierarchy of themes and subthemes, and summarising and synthesising the data. The data credibility was estimated through members checks by sending transcripts to a third independent party (researchers not involved in the study) without knowing the identity of the participants to ensure the reliability of data to review themes and coding system (9). Validity checks included the experiences of the interviewers, clarifying points of view from the interviewees and asking probing questions during interviews to confirm emerged data.

\section{RESULTS}

We thematised data into four main themes. Three of them belong to different OSCE stations which are gathering patient information, examining patients, communicating with patients and the last theme is considered as personal characteristics. Table 1 shows the categories and main themes. Under each theme specific categories were induced as follows:
1. Gathering patient information

a. Theoretical base: Most respondents reported that borderline student has good background knowledge and they emphasised the importance of linking basic science to the patient case. However, he is stereotypical in some areas and is required to fill in the points.

Quote: "Borderline students are theoretically good and logic."

b. Analytical skills: Borderline groups have a good consequence in linking information even if they reach wrong diagnosis, but they don't get in-depth analysis, they may miss to extend their knowledge and elaborate more and may not respond to detailed questions. They know the safety goals, but they may not able to judge the situation.

Quote: "They may miss to review other systems and after gathering information they don't know what to do next."

Quote: "Sometimes they have dissociated ideas."

c. Interpretation skills: They know the indications of the procedures; they interpret results accurately even if they miss some signs; they search for findings that are relevant to the patient case.

Quote: "Even if they miss some findings but they interpret results accurately."

\section{Examining patients}

a. Clinical findings: They need to modify their techniques in a way that delivers them to either positive or negative findings, but they usually do not miss obvious signs and important ones. 
Quote: "There are some signs that we consider them clear pass, most borderline group does not miss these finding."

b. Task fulfillment: They fulfill most of the required items (almost between $60 \%-70 \%$ ), they do not miss important point. Nevertheless, they did not cover the required task thoroughly.

Quote: "Always performance ranges around $60 \%$."

c. Training: They try to manipulate the procedure systemically, however their maneuver (techniques) reflects insufficient repeated practices and need to be adapted with further training.

Quote: "The using of their hands indicates that they perform the task few times, and need to practice more."

3. Communicating with patients

a. Delivering the message (language): They delivers a complete message to patient with simple language. The delivered message is clear and understandable.

Quote: "They deliver the message in understandable language to the patient.”

b. Body language: They have fair body language and eye contact with the patient, and look interested in what the patient says, they listen and do not interrupt patients.

Quote: "They pay attention to what patient is saying." c. Response: They fail to respond to the patient non-verbal communication and patient concern; they need more training to get feedback from patient.

Quote: "They did not pay attention to some patient concerns; they are keen to deliver the message correctly."

\section{Personal characteristics}

a. Organisation: They examine the patient in a systematic manner, do both general and specific; they are organised and follow steps to reach the diagnosis.

Quote: "They follow items of the evaluation checklist."

b. Self-confidence: They usually have less self-confidence. They are slow when performing their tasks and look confused, stressed and hesitant. Most respondents agreed that this is due to less training. However, they admit their limits.

Quote: "They look emotionally instable."

c. Self-efficacy: Borderline groups look uninterested and careless; they want to complete the required task to pass the exam.

Quote: "They take the task lightly; all their concerns is to complete it quickly."

A model for characteristics of borderline group was developed in this study (Figure 2). 
Table 1: The categories and the themes that include the characteristics of borderline group in the different stations of OSCE

\begin{tabular}{ll}
\multicolumn{1}{c}{ Category } & \multicolumn{1}{c}{ Theme } \\
\hline $\begin{array}{l}\text { Theoretical base } \\
\text { Analytical skills } \\
\text { Interpretation skills }\end{array}$ & Gathering patient information \\
\hline $\begin{array}{l}\text { Clinical findings } \\
\text { Training }\end{array}$ & Examining patients \\
Task fulfillment & \\
\hline $\begin{array}{l}\text { Delivering the message } \\
\text { Body language }\end{array}$ & Communicating with patients \\
Response & \\
\hline $\begin{array}{l}\text { Organisation } \\
\text { Self-confidence }\end{array}$ & Personal characteristics \\
\hline
\end{tabular}

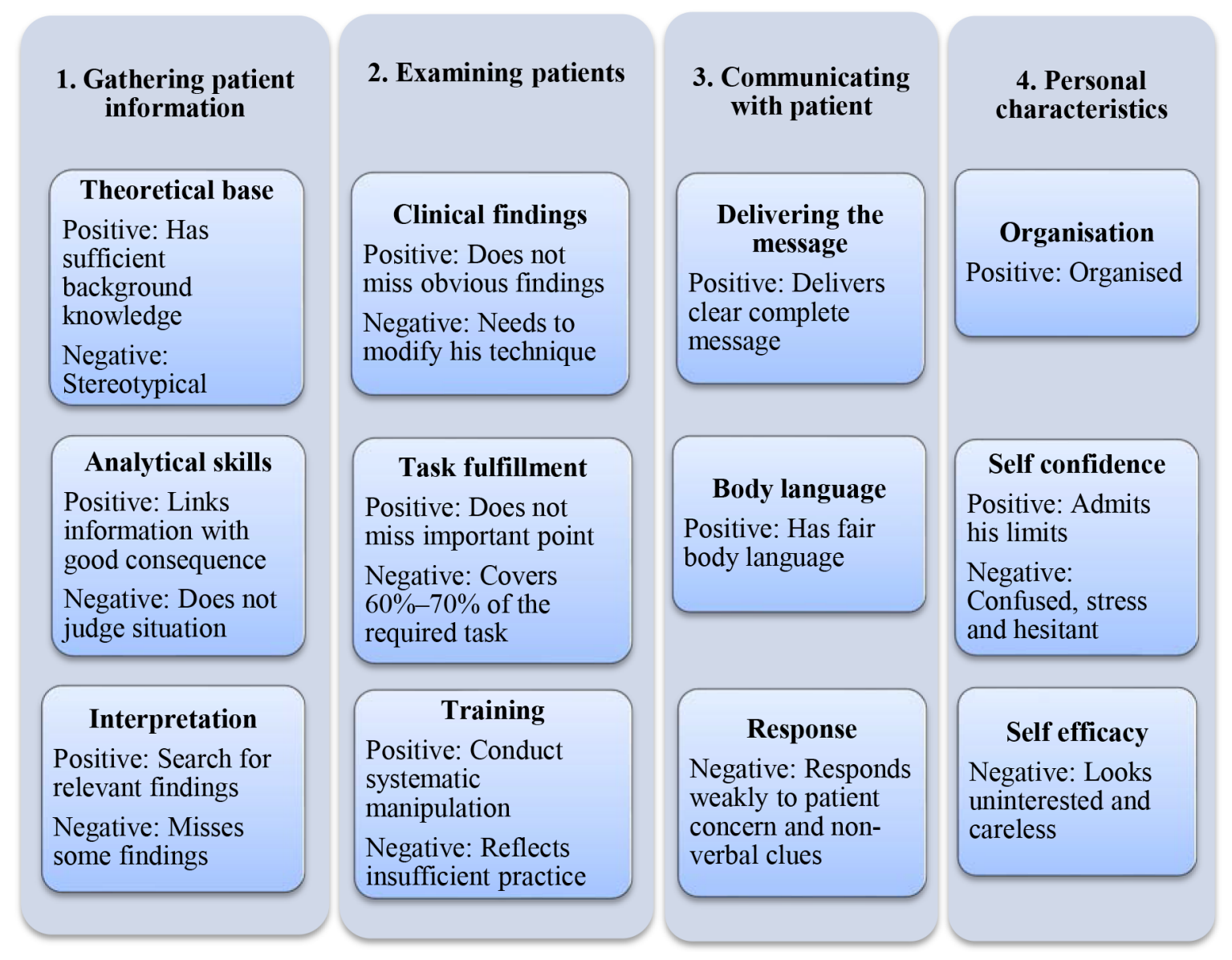

Figure 2: A model for the characteristics of borderline group. 


\section{DISCUSSION}

In this study, we tried to unify definition of minimally competent (borderline) group through setting universal characteristics of minimally competent (borderline) groups in each OSCE stations. However, this study resulted in general performance descriptors such as, gathering patient information; they have sufficient knowledge and fulfill the task, but have a deficient linkage between theory and practice. They interpret results accurately with a partially missing of signs. In examining patients, they examine the patient in a systematic manner. However, they need to modify their techniques to detect either clinical findings, but they usually do not miss obvious signs and important ones. They use their hands inadequately, which reflects insufficient training.

In communicating with patients, they deliver a complete message to the patients with a simple language. The delivered message is clear and understandable. They have fair body language and eye contact with the patient, and look interested in what the patient says, they listen and do not interrupt patients. They fail to respond to patient non-verbal cues and patient concerns; they need more training to get feedback from patient. Their personal characteristics are as follows: They are organised and follow steps to reach the diagnosis. They are lack self-confidence but need to be more careful and faster when completing the required task.

In the present study, it was noticed that all interviewees commented that ranking students as competent, borderline and incompetent is a mentally difficult step because they have no clear criteria for each group. This is comparable with the results of Boursicot's study where they found that setting pass mark differs significantly; and deduced this to difference in conceptualisation of borderline group, as they described the step of conceptualising borderline group as a grey area (10).
In another study, Boursicot found that describing performance as clearly sufficient or insufficient is much easier than describing it as not so bad/not so good or as just enough. In addition, author concluded that making global judgement is better to be articulated for general concept of clinical competence rather than specific items of the clinical competence in the checklist (11). It would be better if judges have clear idea or specific characteristics to indicate borderline group, this could be enhanced if judges share their experiences regarding this concept, through facilitating group discussion beforehand (12).

In most cases, judges have different terminology of minimally competent student including just fair, $50 \%-50 \%$, just adequate...etc. They have different or even contradicting conceptions regarding borderline group; some relate this to foundational knowledge, ability to apply knowledge, reasoning, while others relate their conceptualisation to safe practice. Others think that borderline students are emotional who freak out at the exam situation (13).

Moreover, the same rater may judge the performance of the same student differently when he uses checklist marks in comparison with global grade, and this may be affected by different environment, culture and standard setting team (14). In a qualitative study which was conducted to explore the opinion regarding the standards setting methods, the participants stated that one of the challenges that they faced is defining the borderline group (15). Hence, we need to unify the definition of borderline group in order to make standard setting equivalent regardless of different circumstances.

A previous study replaced the borderline pass grade (50\%) into borderline grade (using a formula referencing the performance of the students in the same domain in other stations), the OSCE included three domains; generic communication skills, physical examination and clinical communication skills. The 
study concluded that defining the borderline group changed the passing rate and obtained satisfaction from the staff (16). The current study demonstrates that defining the characteristics of borderline groups facilitates the pass/fail decision.

As this study revealed, making global judgement for students' performance is a cumbersome mental task and subjective in its nature. It would be better if judges have a clear idea or specific performance descriptors to describe borderline group. This could be enhanced if judges share their experiences regarding this concept, through facilitating group discussion beforehand (10), or if they have a clear performance descriptors of this group.

We thematised emerged data into four themes, three of them belong to the nature of required task in each OSCE station, and one includes themes related to general performance behaviour. In a study by Wilkinson and Harris that describes borderline trainee interns, they suggested three main areas which are basics, clinical task and taking on a professional role. Basic means knowledge, clinical skills, language ability and recognition of common clinical signs (17).

But clinical tasks area means knowledge, skills and attitude required when interacting with a patient to reach a diagnosis or management plan. Finally, taking on a professional role which includes professional attitude such as teamwork, involvement and interpersonal skills. In the previous study, they observed interns during the entire period of training. So, they could comment on professional behaviour. In our research, we studied students' behaviour in the OSCE venue and categorised three themes according to the nature of required task in each station. Boursicot's study reviewed performance descriptor of best, worst and borderline students from five different schools. Based on this observation, five themes were defined such as knowledge, communication skills, interpersonal skills, professionalism and clinical skill proficiency (11).

In this study, we focused on how the student achieved the task, not what he accomplished. In each station, we asked examiner to describe exactly the approach of borderline for student to accomplish the required task. We tried to fill the gap found in the growing body of literature relating to assessors' global judgement on student performance, as previous researchers have found that assessors focus mainly on quantitative methods such as counting the items on the evaluation checklist, and based their judgement on what students do rather than how they do it (18). Unlike additional methods of assessing student clinical skills, OSCE measures student skills in a simulated environment. Communication with patients, patient safety and dealing with unpredictable events are important areas that could be evaluated by OSCE (19).

In a previous study, the pass/fail decision was determined by experts after OSCE for eight pharmacy students. The experts based their decision on the recommendations and management skills. The current study demonstrates the importance of decisionmaking, analytical and interpretation skill of the borderline group (18).

The borderline students are known to be the ones who do not harm the public in their practice. So, identifying the characteristics of this group will be a new contribution for taking a valid decision in clinical exams. Four themes belong to this description are named; gathering patient information, examining patients, communicating with patients and the last theme is considered as personal characteristics. These results will increase the validity and reliability of OSCE. Thus, depending on this method of practitioner assessment will be recommended.

This study has some limitations. It would be better if data were gathered from different medical schools to gain different perspectives. Moreover, observation of 
students' performance in different OSCE stations will add to the emerged themes which were based only on the assessors' viewpoints. For further researches in this area, more studies are needed through structuring a questionnaire for validation of the themes.

\section{CONCLUSION}

Describing minimally competent students (borderline) is an essential step in standard setting, however it is a mentally demanding task. So, having a performance descriptor of borderline group, beforehand, will ease the process.

\section{ETHICAL CONSIDERATIONS}

The study was approved by the ethical committee at the Faculty of Medicine, Suez Canal University. All subjects in this research were informed about the purpose of the study and its relevance to the field of medical education. Only those who accepted to be involved in the study (by signing a consent form) were included. The data collection tools were anonymous. Personal data will not be published under any circumstances. The extracted data from the interview will be used for scientific publishing and educational reform. No harmful consequences are expected from this work.

\section{ACKNOWLEDGEMENTS}

The authors acknowledge the contributions from the participants who shared in this study.

\section{REFERENCES}

1. Kaufman DM, Mann KV, Muijtjens AM, van der Vleuten CP. A comparison of standard-setting procedures for an OSCE in undergraduate medical education. Acad Med. 2000;75(3):267-71. https://doi. org/10.1097/00001888-200003000-00018

2. Munshi FM, Alnemari AH, Alshehri AR, Al-Jifree HM. Standard setting in objective structured clinical examination: assigning a pass/fail cut score. J Health Spec. 2017;5:8-11. https://oi.org/10.4103/24686360.198803

3. Cizek GJ, Bunch MB. The bookmark method. In: Cizek GJ, Bunch MB, editors. Standard setting. Thousand Oaks, CA: Sage Publications; 2007. p. 154-91. https://doi. org/10.4135/9781412985918

4. Homer M, Pell G, Fuller R. Problematizing the concept of the "borderline" group in performance assessments. Med Teach. 2017: 4;39(5):469-75. https://doi.org/10.10 80/0142159X.2017.1296563

5. Elfaki OA, Salih KM. Comparison of two standard setting methods in a medical students MCQs exam in internal medicine. American Journal of Medicine and Medical Sciences. 2015;5(4):164-7.

6. Mortaz Hejri S, Jalili M. Standard setting in medical education: fundamental concepts and emerging challenges. Med J Islam Repub Iran. 2014;28:34.

7. Yudkowsky R, Park YS, Downing SM, editors. Assessment in health professions education. 2nd ed. Routledge e-library; 2019. https://doi. org/10.4324/9781315166902

8. Saldaña J. The coding manual for qualitative researchers. London: Sage; 2015.

9. Guba EG. Criteria for assessing the trustworthiness of naturalistic inquiries. ECTJ. 1981;29:75. https://doi.org/10.1007/ BF02766777 
10. Boursicot K. Setting standards in a professional higher education course: defining the concept of the minimally competent student in performance-based assessment at the level of graduation from medical school. Higher Education Quarterly. 2006;60(1):74-90. https://doi.org/10.1111/ j.1468-2273.2006.00308.x

11. Boursicot KAM, Roberts TE, Pell G. Using borderline methods to compare passing standards for OSCEs at graduation across three medical schools. Med Educ. 2007;41(11):1024-31. https://doi. org/10.1111/j.1365-2923.2007.02857.x

12. Downing SM, Tekian A, Yudkowsky R. Procedures for establishing defensible absolute passing scores on performance examinations in health professions education. Teach Learn Med. 2006;18:50-7. https://doi.org/10.1207/ s15328015tlm1801_11

13. Yeates P, Cope N, Luksaite E, Hassell A, Dikomitis L. Exploring differences in individual and group judgments in standard setting. Med Educ. 2019;53:941-52. https:// doi.org/10.1111/medu.13915

14. Homer M, Pell G, Fuller R. Problematizing the concept of the "borderline" group in performance assessments. Med Teach. 2017;5:469-75. https://doi.org/10.1080/014 2159X.2017.1296563
15. Kamal D, ElAraby S, Kamel MH, Hosny S. Evaluation of two applied methods for standard setting in undergraduate medical programme at the Faculty of Medicine, Suez Canal University. Education in Medicine Journal. 2018;1;10(2):15-25. https://doi. org/10.21315/eimj2018.10.2.3

16. Shulruf B, Adelstein B-A, Damodaran A, Harris P, Kennedy S, O'Sullivan A, et al. Borderline grades in high stakes clinical examinations: resolving examiner uncertainty. BMC Med Educ. 2018;18:272. https://doi.org/10.1186/s12909-018-1382-0

17. Wilkinson TJ, Harris P. The transition out of medical school-a qualitative study of descriptions of borderline trainee interns. Med Educ. 2002;36(5):466-71. https://doi. org/10.1046/j.1365-2923.2002.01209.x

18. Ali M, Pawluk SA, Rainkie DC, Wilby KJ. Pass-fail decisions for borderline performers after a summative objective structured clinical examination. Am J Pharm Educ. 2019;83(2):6849. https://doi.org/10.5688/ ajpe 6849

19. Zayyan M. Objective structured clinical examination: the assessment of choice. Oman Med J. 2011;26(4):219-22. https:// doi.org/10.5001/omj.2011.55 